\title{
NONWANDERING OPERATORS IN BANACH SPACE
}

\author{
LIXIN TIAN, JIANGBO ZHOU, XUN LIU, AND GUANGSHENG ZHONG
}

Received 8 November 2004 and in revised form 27 October 2005

We introduce nonwandering operators in infinite-dimensional separable Banach space. They are new linear chaotic operators and are relative to hypercylic operators, but different from them. Firstly, we show some examples for nonwandering operators in some typical infinite-dimensional Banach spaces, including Banach sequence space and physical background space. Then we present some properties of nonwandering operators and the spectra decomposition of invertible nonwandering operators. Finally, we obtain that invertible nonwandering operators are locally structurally stable.

\section{Introduction}

In the research field of operator, hypercyclic operators and linear chaotic operators have been intensively studied recently. The first observation of hypercyclic operators was by Birkhoff (see [7]). Since then, many researchers discussed this kind of operators (see $[1,2,3,4,5,6,8,9,10,11,12,16,17,18,19,20,22,23,24,35])$. In 1991, for the first time, Godefroy and Shapiro [16] connected the research of hypercyclic operators and linear chaotic operators and pointed out that some hypercyclic operators are chaotic under the definition of Devaney (see $[13,28]$ ). From then on, most hypercyclic operators in the literature have been proved to be chaotic. This implies that almost all hypercyclic operators are linear chaotic. It is well known that linear operators in finite-dimensional linear spaces can not be chaotic but the nonlinear operators may be. Only in infinitedimensional linear spaces can linear operators have chaotic properties. This has attracted wide attention (see $[12,13,15,20,26,27,31]$ ).

While in the research field of differential dynamical system, Axiom $A$ system is an important subject. It requires that the nonwandering set $\Omega(f)$ possesses hyperbolic structure and density of periodic points, where hyperbolic structure is based on Whitney's decomposition and the hyperbolic property of the tangent cluster at each point. However, Axiom $A$ system is restricted in finite-dimensional compact Riemann space. Due to the linear property of operators, its tangent bundle at each point is linear operator itself.

On the basis of the above work, we introduce nonwandering operators in infinitedimensional Banach space, which are the generalization of Axiom $A$ dynamic system but 
different from it. They are new linear chaotic operators and are relative to hypercylic operators, but different from them.

The paper is organized as follows. In Section 2, we list the basic notations and definitions. Then in Section 3, the existence of nonwandering operators on Banach sequence space and physical space is shown. In Section 4, we obtain some basic properties of nonwandering operators. In Section 5, the spectra decomposition of invertible nonwandering operators is completed. Finally, we discuss the local structural stability of invertible nonwandering operators in Section 6.

\section{Basic notations and definitions}

Let $(X,\|\cdot\|)$ be an infinite-dimensional separable Banach space on real number field or complex number field $K$. Let $L(X)$ be the set of all bounded linear operators over $X$. We will refer to $\mathbb{N}, \mathbb{Z}, \mathbb{Q}, \mathbb{R}$, and $\mathbb{C}$ as the sets of positive integers, integers, rational numbers, and the real and complex scalar fields, respectively.

Definition 2.1 (see $[6,18]$ ). Suppose $T \in L(X)$. If there is a vector $x \in X$ such that $\operatorname{Orb}(T, x)=\left\{x, T x, T^{2} x, \ldots\right\}$ is dense in $X$, then call $x$ a hypercyclic vector and $T$ a hypercyclic operator.

Definition 2.2 (see $[3,13])$. Suppose $T \in L(X)$, call $T$ a linear chaotic operator or a linear chaotic map if it satisfies the following two conditions:

(1) $T$ is topologically transitive, that is, $T$ has a dense orbit in $X$;

(2) the set of periodic points $\operatorname{Per}(T)$ for $T$ is dense in $X$.

Remark 2.3. The definition of chaotic map in the sense of Devaney needs another condition:

(3) $T$ has a sensitive dependence on initial conditions (see [3]). In fact, conditions (1) and (2) imply condition (3) (see [13]). Thus condition (3) can be omitted from Definition 2.2.

Because of the complexity of infinite-dimensional dynamic systems, research of their chaotic properties is usually changed to the study of attractors and inertial manifolds (see [21]). Attractors and inertial manifolds in infinite-dimensional linear space are restricted to be closed invariant linear subspaces (see [21]). So we give the definition of nonwandering operators on closed invariant linear subspace.

Definition 2.4. Suppose $E \subset X$ is a closed linear subspace of $X$, and $E_{1} \subset E, E_{2} \subset E$ are also closed linear subspaces in $X$. For arbitrary $x \in E$, if there is a unique decomposition such that $x=x_{1}+x_{2}, x_{1} \in E_{1}, x_{2} \in E_{2}, E_{1} \cap E_{2}=\{0\}$, then $E$ is called the direct sum of $E_{1}$ and $E_{2}$, and written as $E=E_{1} \oplus E_{2}$, where $\oplus$ represents direct sum.

Definition 2.5. Suppose $T \in L(X)$. (1) Assume that there exists a closed subspace $E \subset X$, which has hyperbolic structure: $E=E^{u} \oplus E^{s}, T E^{u}=E^{u}, T E^{s}=E^{s}$, where $E^{u}, E^{s}$ are closed subspaces. In addition, there exist constants $\tau(0<\tau<1)$ and $c>0$, such that for any $\xi \in E^{u}, k \in \mathbb{N},\left\|T^{k} \xi\right\| \geq c \tau^{-k}\|\xi\|$, and for any $\eta \in E^{s}, k \in \mathbb{N},\left\|T^{k} \eta\right\| \leq c \tau^{k}\|\eta\|$. (2) Assume also that $\operatorname{Per}(T)$ is dense in $E$. Then $T$ is said to be a nonwandering operator relative to $E$. 
Remark 2.6. (1) $T$ may be invertible or not. When $T$ is invertible, the spectral property of nonwandering operators is different from that of hypercyclic operators (see Theorem 4.2), but when $T$ is not invertible, the case is much complicated. We give an example for such case. (See Remark 3.5.)

(2) If $T$ is a nonwandering operator, then $\operatorname{Per}(T) \cap E=\Phi$. In fact, we can easily get it from the hyperbolic structure of $E$.

(3) Because $T$ is a linear operator, the tangent bundle at each point in $E$ is $T$ itself. Therefore, the definition of nonwandering operators is the natural generalization of Axiom $A$ dynamic system in finite-dimensional differentiable dynamical systems to infinitedimensional space. And these operators are meaningful.

Definition 2.7. Suppose $T \in L(X)$ and $\left\{e_{i}\right\}_{1}^{\infty}$ is a basis in $X$, then $T$ is called a unilateral backward shift operator relative to $\left\{e_{i}\right\}_{1}^{\infty}$ if $T e_{n}=e_{n-1}(n>1)$ and $T e_{1}=0$.

Definition 2.8. Let $E \subset X$ be a closed linear subspace of $T$. If there exist countable closed invariant subsets $E_{1}, E_{2}, \ldots, E_{n}, \ldots$ (any two of them are never intersected) such that $E=$ $\bigcup_{i=1}^{\infty} E_{i}$, and for arbitrary nonempty open subsets $U, V \subset E_{i}$, there exists $n \in \mathbb{N}$, such that $T^{n} U \cap V \neq \Phi$, then call it the spectra decomposition of $T$ for $E$, and $E_{1}, E_{2}, \ldots$, $E_{n}, \ldots$ are called the basic sets.

Definition 2.9. Suppose that $\left(X,\|\cdot\|_{1}\right),\left(Y,\|\cdot\|_{2}\right)$ are two Banach spaces. $f: X \rightarrow Y$ is called a Lipschitz mapping if there exists $\alpha>0$, such that for any $x, y \in X, \| f(x)-$ $f(y)\left\|_{2} \leq \alpha\right\| x-y \|_{1}$, where the smallest $\alpha$ is written as $\operatorname{Lip}(f)$.

Definition 2.10. Let $T_{i}: X_{i} \rightarrow X_{i}(i=1,2)$ be two operators in Banach space $X_{i}$. If there exists a homeomorphism $\varphi: X_{1} \rightarrow X_{2}$ such that $\varphi \circ T_{1}=T_{2} \circ \varphi$, then $T_{1}$ is called topologically conjugate to $T_{2}$.

Definition 2.11. Let $T \in L(X)$ be a nonwandering operator relative to $E$. $T$ is called locally structural stable in $L(X)$ if there is a neighborhood $U$ of $T$ and a nonempty open subset $V \subset E$, such that for each linear operator $S \in U, S$ is topologically conjugate to $T$ on $V$.

\section{Existence of nonwandering operators in Banach sequence space}

\subsection{Nonwandering operators in Banach sequence space.}

Proposition 3.1 (see [19]). Let X be a Banach sequence space on countable infinite index set, consider the following assertions:

(1) $\left(e_{i}\right)_{i \in I}$ is an unconditional basis;

(2) $\left(e_{i}\right)_{i \in I}$ is a basis in some ordering, and if $\left(x_{i}\right) \in X$, then also $\left(\varepsilon_{i} x_{i}\right) \in X$ whenever each $\varepsilon_{i}$ is either 0 or 1 ;

(3) $\left(e_{i}\right)_{i \in I}$ is a basis in some ordering, and if $\left(x_{i}\right) \in X$, then also $\left(c_{i} x_{i}\right) \in X$ whenever $\left(c_{i}\right)$ is a bounded order of scalars.

Then the following implications hold: (1) $\Leftrightarrow(2) \Rightarrow(3)$.

Theorem 3.2. Each infinite-dimensional separable Banach sequence space with an unconditional basis supports a nonwandering operator. 
Proof. Let $X$ be an infinite-dimensional separable Banach sequence space, in which $\left\{e_{i}\right\}_{1}^{\infty}$ is an unconditional basis, then for the unilateral backward shift operator $T$ on $X, \lambda T$ is a nonwandering operator on $X$ whenever $\sqrt{2}<\lambda<2$.

In fact, we can construct a closed invariant subspace $E \subset X$ such that $E$ has hyperbolic structure. Let $l=\lambda / 2$, then we have $0<|l|<1$. Suppose $y_{0}=\sum_{i=1}^{\infty} b_{i} e_{i}$ such that $\lambda T y=l y$, then we get a vector $y_{0}=\left\{b_{1},(1 / 2) b_{1},(1 / 2)^{2} b_{1}, \ldots\right\}$. Let $E^{s}=\overline{\operatorname{span}\left\{y_{0}\right\}}$, then $E^{s}$ is a closed invariant subspace of eigenvectors corresponding to the eigenvalue $l=\lambda / 2$ for $\lambda T$. Thus, for each $x \in E^{s}$, there is $x=m y_{0},\|(\lambda B) x\|=\left\|\lambda m B y_{0}\right\|=|l|\left\|m y_{0}\right\|=|l|\|x\|$, where $0<$ $|l|<1$. Let $k=1 / l=2 / \lambda$. Suppose $y_{1}=\sum_{i=1}^{\infty} c_{i} e_{i}$, which satisfies $\lambda T y=k y$, then we get a vector $y_{1}=\left\{b_{1},\left(2 / \lambda^{2}\right) b_{1},\left(2 / \lambda^{2}\right)^{2} b_{1}, \ldots\right\}$. Let $E^{u}=\overline{\operatorname{span}\left\{y_{1}\right\}}$, then $E^{u}$ is a closed invariant subspace of eigenvectors corresponding to eigenvalue $k=2 / \lambda$ for $\lambda T$. Thus, for each $y \in$ $E^{u}$, there is $y=m y_{1},\|(\lambda T) y\|=\left\|(\lambda T) m y_{1}\right\|=k\left\|m y_{1}\right\|=k\|y\|$, where $|k|>1$. Let $E=$ $E^{u} \oplus E^{s}$ and take $\tau(|l|<\tau<1)$, then for each $\xi \in E^{u}, k \in \mathbb{N},\left\|T^{k} \xi\right\| \geq \tau^{-k}\|\xi\|$, and for each $\eta \in E^{s}, k \in \mathbb{N},\left\|T^{k} \eta\right\| \leq \tau^{k}\|\eta\|$.

Next, we prove $\operatorname{Per}(\lambda T)$ is dense in $X$. For each $n \in \mathbb{N}, \lambda T(|\lambda|>1)$ has $n$-period points, such as $x=\left\{x_{1}, x_{2}, \ldots, x_{n}, x_{1} / \lambda^{n}, x_{2} / \lambda^{n}, \ldots, x_{n} / \lambda^{n}, x_{1} / \lambda^{2 n}, x_{2} / \lambda^{2 n}, \ldots, x_{n} / \lambda^{2 n}, \ldots\right\}$, where $\left\{x_{i}\right\}_{i=1}^{n}$ $\subset \mathbb{R}$. Let $\bar{y}=\left(y_{n}\right) \in X$ be an $n_{0}$-period point for $\lambda T$, then there exist $n_{0} \in \mathbb{N}$ and $i \in \mathbb{N}$, such that $(\lambda T)^{n_{0}} \bar{y}=\bar{y}$ and $y_{i} \neq 0$. By Proposition 3.1, for each $i \in \mathbb{N}$ and $n_{0} \in \mathbb{N}$ the series

$$
y^{\left(i, n_{0}\right)}:=\lambda^{i} \sum_{k=0}^{\infty} \lambda^{-\left(i+k n_{0}\right)} e_{i+k n_{0}}
$$

converge in $X$. Moreover, if $n_{0} \geq i$, then

$$
(\lambda T)^{n_{0}} y^{\left(i, n_{0}\right)}=y^{\left(i, n_{0}\right)} .
$$

Therefore, each $y^{\left(i, n_{0}\right)}\left(i \leq n_{0}\right)$ is a periodic point for $\lambda T$.

Now we will prove that $\lambda T$ has dense set of periodic points. For each $z \in \operatorname{span}\left\{e_{n}: n \in\right.$ $\mathbb{N}\}$, we suppose $z=\sum_{i=1}^{m} z_{i} e_{i}$ and

$$
\left|z_{i} \lambda^{i}\right| \leq \mathbb{C}, \quad i=1,2, \ldots, m
$$

where $\mathbb{C}$ is a constant. Since $\left(e_{n}\right)$ is an unconditional basis and the series $\sum_{n=1}^{\infty} \lambda^{-n} e_{n}$ converge in $X$, there exist $n_{0} \geq m$, such that

$$
\left\|\sum_{n=n_{0}+1}^{\infty} \varepsilon_{n} \lambda^{-n} e_{n}\right\|<\frac{\varepsilon}{c m},
$$

where $\left(\varepsilon_{n}\right)$ takes 0 or 1 . By (3.2), $y=\sum_{i=1}^{m} z_{i} y^{\left(i, n_{0}\right)}$ is a periodic point for $T$ in $X$, and

$$
\begin{aligned}
\|y-z\| & =\left\|\sum_{i=1}^{m} z_{i}\left(y^{\left(i, n_{0}\right)}-e_{i}\right)\right\|=\left\|\sum_{i=1}^{m}\left(z_{i} \lambda^{i}\right) \sum_{k=1}^{\infty} \lambda^{-\left(i+k n_{0}\right)} e_{i+k n_{0}}\right\| \\
& \leq \sum_{i=1}^{m}\left\|\left(z_{i} \lambda^{i}\right) \sum_{k=1}^{\infty} \lambda^{-\left(i+k n_{0}\right)} e_{i+k n_{0}}\right\| .
\end{aligned}
$$


Then by (3.4), we have $\|y-z\| \leq \varepsilon$, namely, there exists a periodic point $y$ arbitrarily close to $z$. We obtain that $\operatorname{Per}(\lambda T)$ is dense in $X$, and so is in $E$. Thus $\lambda T$ is a nonwandering operator relative to $E$.

Remark 3.3. In this theorem, closed invariant subspaces $E^{s}, E^{u}$ are finite dimensional. In the next, we present an example in physics, in which $E^{s}, E^{u}$ are infinite dimensional.

3.2. Nonwandering operator in physical background system. There are examples for hypercyclic and linear chaotic operators in physics (see [14, 20, 26, 31]). Similarly, nonwandering operators can occur in systems of concrete physical background. Consider a very small frictionless mass-spring system whose evolution is determined by Schrodinger equation:

$$
i \hbar \dot{\psi}=-\frac{\hbar}{2 m} \psi^{\prime \prime}+\frac{k}{2} x^{2} \psi
$$

with displacement $x$, mass $m$, stiffness $k$, natural frequency $\omega=\sqrt{k / m}$, and wave function $\psi$ to be determined in the complex separable Hilbert space $X=L^{2}(-\infty, \infty)$. It is easy to see that (3.6) can be rewritten as

$$
i \dot{\psi}=-\psi^{\prime \prime}+x^{2} \psi
$$

The stationary states $\psi$ satisfy

$$
\psi^{\prime \prime}-x^{2} \psi=-\lambda \psi
$$

and so do the polynomial

$$
\psi_{n}=e^{-x^{2} / 2} \frac{H_{n}(x)}{\sqrt{\sqrt{\pi} 2^{n} n !}},
$$

where $H_{n}(x)=(-1)^{n} e^{x^{2}}\left(d / d x^{n}\right) e^{x^{2}}$ is the $n$-Hermite polynomial.

Noting that $H_{n}^{\prime}(x)=2 n H_{n-1}(x)$, we have the following iteration:

$$
B \psi_{n}=\frac{1}{\sqrt{2}}\left(x+\frac{d}{d x}\right) \psi_{n}=\sqrt{n} \psi_{n-1} .
$$

The unobservable differential (annihilation, lowering) operator $B$ of (3.10) is an unbounded densely defined and weighted backward shift operator in $X=L^{2}(-\infty,+\infty)$.

The natural space for the quantum harmonic oscillator is the Banach space Fof all rapidly decreasing functions, that is,

$$
F=\left\{\phi \in L^{2}(-\infty,+\infty), \phi=\sum_{n=0}^{\infty} c_{n} \psi_{n}, \sum_{n=0}^{\infty}\left|c_{n}\right|^{2}(n+1)^{r}<\infty, \forall r \geq 0\right\} .
$$

The norm $\|\cdot\|$ of $F$ is defined as

$$
\forall \phi=\sum_{n=0}^{\infty} c_{n} \psi_{n} \in F, \quad\|\phi\|=\left[\sum_{n=0}^{\infty}\left|c_{n}\right|^{2}(n+1)^{r}\right]^{1 / 2} \quad(r \geq 0)(\text { see [26]). }
$$


Under the norm, $B$ is continuous on space $F$ (see [26]). So $B$ is bounded operator on space $F$.

Theorem 3.4. The annihilation operator B on Banach space $F$ is a nonwandering operator.

Proof. For each $\lambda \in \mathbb{R}$, it is easy to obtain that $\phi_{\lambda}=\sum_{n=0}^{\infty}\left(\lambda^{n} / \sqrt{n !}\right) \psi_{n}$ is an eigenvector corresponding to $\lambda$. Let $E_{1}=\left\{\phi=\sum_{i=0}^{\infty} a_{i} \phi_{\lambda_{i}}=\sum_{n=0}^{\infty}\left(\sum_{i=0}^{\infty} a_{i} \lambda_{i}^{n} / \sqrt{n !}\right) \psi_{n} \mid B \phi_{\lambda_{i}}=\lambda_{i} \phi_{\lambda_{i}}, \forall n \in\right.$ $\mathbb{N}, \sum_{i=0}^{\infty} a_{i} \lambda_{i}^{n}<\infty$, where $\left.\lambda_{i} \geq 2, i=0,1,2, \ldots\right\}, E^{u}=\overline{E_{1}}$, then $E^{u} \subset F$ is a closed subspace.

For each $\phi=\sum_{i=0}^{\infty} a_{i} \phi_{\lambda_{i}}=\sum_{n=0}^{\infty}\left(\sum_{i=0}^{\infty} a_{i} \lambda_{i}^{n} / \sqrt{n !}\right) \psi_{n} \in E^{u}$ and $k \in \mathbb{N}$, we have

$$
\begin{aligned}
\left\|B^{k} \phi\right\|^{2} & =\left\|\sum_{n=0}^{\infty}\left(\frac{\sum_{i=0}^{\infty} a_{i} \lambda_{i}^{k} \lambda_{i}^{n}}{\sqrt{n !}}\right) \psi_{n}\right\|^{2}=\sum_{n=0}^{\infty} \frac{\left(\sum_{I=0}^{\infty} a_{i} \lambda_{i}^{k} \lambda_{i}^{n}\right)^{2}}{n !}(n+1)^{r} \\
& \geq 2^{2 k}\left[\sum_{n=0}^{\infty} \frac{\left(\sum_{i=0}^{\infty} a_{i} \lambda_{i}^{n}\right)^{2}}{n !}(n+1)^{r}\right]=2^{2 k}\|\phi\|^{2}, \quad \forall r \geq 0 .
\end{aligned}
$$

By (3.13) we have $\left\|B^{k} \phi\right\| \geq 2^{k}\|\phi\|$. Let $E_{2}=\left\{\psi=\sum_{i=0}^{\infty} b_{i} \phi_{\tau_{i}}=\sum_{n=0}^{\infty}\left(\sum_{i=0}^{\infty} b_{i} \tau_{i}^{n} / \sqrt{n !}\right) \psi_{n} \mid\right.$ $B \phi_{\tau_{i}}=\tau_{i} \phi_{\tau_{i}}$, for each $n \in \mathbb{N}, \sum_{i=0}^{\infty} b_{i} \tau_{i}^{n}<\infty$, where $\left.0<\tau_{i} \leq 1 / 2, i=0,1,2, \ldots\right\}$ and $E^{s}=$ $\overline{E_{2}}$. Then $E^{s} \subset F$ is a closed subspace and for each $k \in \mathbb{N}, \psi \in E^{s}$, we have $\left\|B^{k} \psi\right\| \leq$ $(1 / 2)^{k}\|\psi\|$.

In the following we will prove that $E^{u}$ and $E^{s}$ are invariant under the operator $B$.

On one hand, for each $\phi \in E^{u}, \phi=\sum_{i=0}^{\infty} a_{i} \phi_{\lambda_{i}}=B \sum_{i=0}^{\infty}\left(a_{i} / \lambda_{i}\right) \phi_{\lambda_{i}} \in B E^{u}, E^{u} \subset B E^{u}$. On the other hand, for each $\psi \in B E^{u}$, there exists $\phi=\sum_{i=0}^{\infty} a_{i} \phi_{\lambda_{i}} \in E^{u}$, such that $B \phi=\psi$, then $\psi=B\left(\sum_{i=0}^{\infty} a_{i} \phi_{\lambda_{i}}\right)=\sum_{i=0}^{\infty}\left(a_{i} \lambda_{i}\right) \phi_{\lambda_{i}} \in E^{u}$, so $B E^{u} \subset E^{u}$. In conclusion, we get $B E^{u}=E^{u}$. Similarly, $B E^{s}=E^{s}$ holds.

Finally we prove that $\operatorname{Per}(B)$ is dense in $F$. Let $\phi_{\lambda}$ be an eigenvector corresponding to $\lambda$, where $\lambda$ are roots of unity. Then $\phi_{\lambda}$ initiate periodic orbits of $B$. Thus $\phi_{\lambda}$ are dense in $F$. If not, then there is some function $\alpha=\sum_{n=0}^{\infty} a_{n} \psi_{n}$ in $F$ which is orthogonal to each such $\phi_{\lambda}$, that is,

$$
\left(\phi_{\lambda}, \alpha\right)=\sum_{n=0}^{\infty} \frac{\overline{a_{n}} \lambda^{n}}{\sqrt{n !}} \equiv 0 .
$$

But the zeros of analytic functions are isolated, so $\operatorname{Per}(B)$ is dense in $F$, thus $B$ is a nonwandering operator.

Remark 3.5. (1) The annihilation operator $B$ is not an invertible nonwandering operator. Here $\sigma(B) \bigcap \partial D \neq \Phi$, where $\sigma(B)$ is the spectrum of $B$, and $\partial D$ is unit circle. In fact, we have $\sigma(B)=\mathbb{C}$.

(2) Although nonwandering operators are relative to hypercyclic operators, some hypercyclic operators are not nonwandering operators. For example, the "Bergman" backward shift operator $B$ (see $[11$, Section3.8]), corresponding to weight sequence $\beta(n)=$ $1 / n+1$, is hypercyclic (see [34]), but is not a nonwandering operator because it does not possess dense set of periodic points (see [34]).

(3) There exists a nonwandering operator, but it is not hypercyclic. For example, let $(X,\|\cdot\|)$ be a Banach space, and let $B$ be a nonwandering operator relative to $E=E^{s} \oplus E^{u}$ given in Theorem 3.4. But $B$ is not a hypercyclic operator on space $E$. Otherwise, there 
exists a vector $x \in E$ such that $\left\{B^{n} x: n=0,1,2, \ldots\right\}$ is dense in $E$ (see Definition 2.1). Suppose $x=x_{1}+x_{2}, x_{1} \in E^{u}, x_{2} \in E^{s}$. For each $y \in E$, there exist $n_{i}$ such that

$$
\begin{aligned}
\left\|B^{n_{i}} x-y\right\| & =\left\|B^{n_{i}} x_{1}+\left(B^{n_{i}} x_{2}-y\right)\right\| \geq\left\|B^{n_{i}} x_{1}\right\|-\left\|B^{n_{i}} x_{2}-y\right\| \\
& \geq\left\|B^{n_{i}} x_{1}\right\|-\left\|B^{n_{i}} x_{2}\right\|-\|y\| \geq c \tau^{-n_{i}}\left\|x_{1}\right\|-c \tau^{n_{i}}\left\|x_{2}\right\|-\|y\| \stackrel{n_{i} \rightarrow+\infty}{\longrightarrow} \infty .
\end{aligned}
$$

Thus it is contrary to the density of the orbit under $B$, and then $B$ is not hypercyclic on $E$.

\section{Properties of nonwandering operators}

Proposition 4.1. Suppose $T \in L(X,\|\cdot\|)$ and $E \subset X$ is a closed subspace, then $T$ is a nonwandering operator relative to $E$ if and only if the following conditions hold: (1) $E=$ $E^{u} \oplus E^{s}, T E^{u}=E^{u}, T E^{s}=E^{s}$, and there exists some norm $|\cdot|$, which is equivalent to $\|\cdot\|$, such that $\left|T_{u}\right|=\left.|T|_{E^{u}}|>1,| T_{s}|=| T\right|_{E^{s}} \mid<1$. (2) $\operatorname{Per}(T)$ is dense in $E$.

$B y$ [37], the following spectral properties of nonwandering operators are obtained.

Theorem 4.2. Let $T \in L(X)$ be invertible, and let $E \subset X$ be a closed subspace. Then $T$ is an invertible nonwandering operator relative to $E$ if and only if (1) its spectral set $\sigma(T)$ does not intersect the unit circle on complex plane, that is, $\sigma(T) \bigcap \partial D=\Phi ;(2) \operatorname{Per}(T)$ is dense in E.

Remark 4.3. (1) Theorem 4.2 requires that $T$ is invertible; otherwise, Theorem 4.2 may be not true, see Remark 3.5.

(2) For hypercyclic operator $T \in L(X)$, we have $\sigma(T) \bigcap \partial D \neq \Phi$ (see [24]). However, the above Theorem 4.2 shows that nonwandering operators differ from hypercyclic operator when it is an invertible operator. Hence they have completely different properties, although they are actually both connected to linear chaotic operators (see Remark 3.5).

Ansari [1] obtained the following result: if $T$ is a hypercydic operator on complex separable Banach space, then so is $T^{m}$; moreover, $T$ and $T^{m}$ have the same hypercyclic vectors. Similarly we obtain the following results for nonwandering operators.

Theorem 4.4. Suppose $T \in L(X)$ and $T$ is an invertible nonwandering operator relative to closed subspace $E$, then so are $T^{m}$ and $T^{-m}$ for each $m \in \mathbb{N}$.

Proof. Obviously, $T^{m}$ and $T^{-m}$ satisfy condition (1) in Definition 2.5. We have that periodic points of $T$ are also the ones of $T^{m}$ and $T^{-m}$. Because $\operatorname{Per}(T)$ is dense in $E$, then $\operatorname{Per}\left(T^{m}\right)$ and $\operatorname{Per}\left(T^{-m}\right)$ are also dense in $E$. Therefore $T^{m}$ and $T^{-m}$ are also nonwandering operators relative to $E$.

Theorem 4.5. Let $(X,\|\cdot\|)$ be an infinite-dimensional separable Banach space, and let $E_{1}, E_{2}$ be closed subspaces in $X$ and $E_{1} \cap E_{2}=\{0\}$. If the restrictions $\left.T\right|_{E_{1}},\left.T\right|_{E_{2}} \in L(X)$ are invertible nonwandering operators relative to $E_{1}, E_{2}$, respectively, then $\left.T\right|_{E}$ is a nonwandering operator relative to $E=E^{u} \oplus E^{s}$.

Proof. Since $\left.T\right|_{E_{i}}(i=1,2)$ is a nonwandering operator relative to $E_{i}$, then $E_{i}$ has hyperbolic structure: $E_{i}=E_{i}^{u} \oplus E_{i}^{s}, T E_{i}^{u}=E_{i}^{u}, T E_{i}^{s}=E_{i}^{s}$, where $E_{i}^{s}, E_{i}^{u}$ are also closed subspaces. Furthermore, there exist $0<\tau_{i}<1$ and constant $c_{i}>0$, such that, for each $\xi_{i} \in E_{i}^{u}, \eta_{i} \in E_{i}^{s}$, $k \in \mathbb{N}$, we have $\left\|T^{-k} \xi_{i}\right\| \leq c_{i} \tau_{i}^{k}\left\|\xi_{i}\right\|,\left\|T^{k} \eta_{i}\right\| \leq c_{i} \tau_{i}^{k}\left\|\eta_{i}\right\|$, and $\operatorname{Per}\left(\left.T\right|_{E_{i}}\right)$ is dense in $E_{i}$. 
Let $E^{u}=E_{1}^{u} \oplus E_{2}^{u}$, we define the following norm on $E^{u}$ : for all $x \in E^{u}, x=x_{1}+x_{2}, x_{1} \in$ $E_{1}^{u}, x_{2} \in E_{2}^{u},\|x\|_{0}=\max \left\{\left\|x_{1}\right\|,\left\|x_{2}\right\|\right\}$, then $\|\cdot\|_{0}$ is equivalent to $\|\cdot\|$ (see Lemma 5.3). Namely, for all $x \in E^{u}$, there exist constants $c_{i}>0(i=3,4)$, such that $c_{4}\|x\| \leq\|x\|_{0} \leq$ $c_{3}\|x\|$.

For each $x \in E^{u}, x=x_{1}+x_{2}, x_{1} \in E_{1}^{u}, x_{2} \in E_{2}^{u}$, we have $\left\|T^{-k} x\right\|=\left\|T^{-k}\left(x_{1}+x_{2}\right)\right\| \leq$ $\left\|T^{-k} x_{1}\right\|+\left\|T^{-k} x_{2}\right\| \leq c_{1} \tau_{1}^{k}\left\|x_{1}\right\|+c_{2} \tau_{2}^{k}\left\|x_{2}\right\| \leq c_{1} \tau_{1}^{k}\|x\|_{0}+c_{2} \tau_{2}^{k}\|x\|_{0} \leq c_{1} c_{3} \tau_{1}^{k}\|x\|+$ $c_{2} c_{3} \tau_{2}^{k}\|x\|$. Let $c=\max \left\{c_{1} c_{3}, c_{2} c_{3}\right\}, \tau=\max \left\{\tau_{1}, \tau_{2}\right\}$, then $c>0,0<\tau<1$, and for each $x \in E^{u}, k \in \mathbb{N}$, then $\left\|T^{-k} x\right\| \leq 2 c \tau^{k}\|x\|$ holds. Let $E^{s}=E_{1}^{s} \oplus E_{2}^{s}$, then for each $y \in E^{s}, k \in$ $\mathbb{N}$, we get $\left\|T^{k} y\right\| \leq 2 c \tau^{k}\|y\|$. Therefore, $E=E^{u} \oplus E^{s}, T E^{u}=T E_{1}^{u} \oplus T E_{2}^{u}=E_{1}^{u} \oplus E_{2}^{u}=E^{u}$, $T E^{s}=T E_{1}^{s} \oplus T E_{2}^{s}=E_{1}^{s} \oplus E_{2}^{s}=E^{s}$. Since $\operatorname{Per}\left(\left.T\right|_{E_{1}}\right), \operatorname{Per}\left(\left.T\right|_{E_{2}}\right)$ are dense in $E_{1}, E_{2}$, respectively, $\operatorname{Per}\left(\left.T\right|_{E}\right)$ is dense in $E_{1} \oplus E_{2}=E^{u} \oplus E^{s}=E$. Thus $\left.T\right|_{E}$ is a nonwandering operator relative to $E$.

\section{Spectra decomposition of nonwandering operators}

In this section, we give the spectra decomposition of invertible nonwandering operators $T$ relative to infinite-dimensional closed subspace.

Theorem 5.1. Suppose $T \in L(X)$ is an invertible nonwandering operator relative to infinitedimensional closed subspace $E$, then there exist closed disjoint nonempty subsets $E_{i} \subset X$ ( $i \in$ $\mathbb{N})$, such that $E=\bigcup_{i=1}^{\infty} E_{i}$, and for arbitrary nonempty open sets $U, V \subset E_{i}$, there exists $n \in$ $\mathbb{N}$, such that $T^{n} U \cap V \neq \Phi$.

In order to prove the theorem, we firstly introduce the following notations. For $y \in X$, let

$$
\begin{gathered}
W_{\eta}^{u}(y)=\left\{x \in E \mid\left\|T^{k}(y-x)\right\|>\eta, \lim _{k \rightarrow+\infty}\left\|T^{-k}(y-x)\right\|=0\right\}, \\
W_{\eta}^{s}(y)=\left\{x \in E \mid\left\|T^{k}(y-x)\right\|<\eta, \lim _{k \rightarrow+\infty}\left\|T^{k}(y-x)\right\|=0\right\}, \\
W^{u}(y)=\left\{x \in E \mid \lim _{k \rightarrow+\infty}\left\|T^{-k}(y-x)\right\|=0\right\}, \\
W^{s}(y) E=\left\{x \in E \mid \lim _{k \rightarrow+\infty}\left\|T^{k}(y-x)\right\|=0\right\}, \\
W_{p}=W^{u}(p, T) \bigcap E, \quad X_{p}=\overline{W_{p}}, \quad B_{\eta}(s)=\{y \in E \mid\|y-s\|<\eta\},
\end{gathered}
$$

where $s \in E, p \in \operatorname{Per}(T)$. Thus we have $X_{p} \subset B_{\eta}\left(W_{p}\right) \subset B_{\eta}\left(X_{p}\right)$. Now we need the following lemmas.

Lemma 5.2. Let $X$ be a Banach space, $\|\cdot\|$ and let $\|\cdot\|_{0}$ be two different norms on it. If there exist some constant $a>0$, such that $\|x\| \leq a\|x\|_{0}$, then $\|\cdot\|$ is equivalent to $\|\cdot\|_{0}$, that is, there exist constants $c_{i}>0(i=1,2)$, such that $c_{2}\|x\| \leq\|x\|_{0} \leq c_{1}\|x\|$.

Proof. By equivalent norm theorem and Banach inverse operator theorem, we can easily obtain this result.

Lemma 5.3. Let $E=E^{u} \oplus E^{s}$ be closed subspace with the norm $\|\cdot\|$, we define a new norm $\|\cdot\|_{0}$ in $E$ as follows: for each $x \in E, x=\xi+\eta, \xi \in E^{u}, \eta \in E^{s},\|x\|_{0}=\max \{\|\xi\|,\|\eta\|\}$, then 
$\|\cdot\|_{0}$ is equivalent to $\|\cdot\|$, namely, there exist $c_{i}>0(i=1,2)$ such that $c_{2}\|x\| \leq\|x\|_{0} \leq$ $c_{1}\|x\|$.

Proof. we can easily prove that $\left(X,\|\cdot\|_{0}\right)$ is a Banach space. Furthermore, for all $x \in X$, we have $\|x\| \leq 2\|x\|_{0}$. Then by Lemma 5.2, Lemma 5.3 holds.

Lemma 5.4. For $\varepsilon>0$ small enough, if $\|y-z\|<\delta<\varepsilon, y, z \in E$, then $W_{\varepsilon}^{u}(y)$ and $W_{\varepsilon}^{s}(z)$ have a unique common point.

Proof. Let $y=\xi_{0}+\eta_{0}, z=\xi_{1}+\eta_{1}, \xi_{0}, \xi_{1} \in E^{u}, \eta_{0}, \eta_{1} \in E^{s}$. Suppose $x=\eta_{0}+\xi_{1}$, if $\|y-z\|<$ $\delta<\varepsilon$, then we get

$$
\begin{gathered}
\left\|T^{-k}(x-y)\right\|=\left\|T^{-k}\left(\xi_{1}-\xi_{0}\right)\right\| \leq c \tau^{k}\left\|\xi_{1}-\xi_{0}\right\| \longrightarrow 0 \quad(k \longrightarrow \infty), \\
\left\|T^{k}(x-z)\right\|=\left\|T^{k}\left(\eta_{1}-\eta_{0}\right)\right\| \leq c \tau^{k}\left\|\eta_{1}-\eta_{0}\right\| \longrightarrow 0 \quad(k \longrightarrow \infty) .
\end{gathered}
$$

Hence $x \in W_{\varepsilon}^{u}(y) \cap W_{\varepsilon}^{s}(z)$. We conclude that $x$ is unique. If not, there exists another point $x_{0} \in W_{\varepsilon}^{u}(y) \cap W_{\varepsilon}^{s}(z)$. Let $x_{0}=x_{1}+x_{2}, x_{1} \in E^{u}, x_{2} \in E^{s}$, then by Lemma 5.3, we have

$$
\begin{aligned}
x-x_{0} & =\left(\xi_{1}-x_{1}\right)+\left(\eta_{0}-x_{2}\right), \quad \xi_{1}-x_{1} \in E^{u}, \eta_{0}-x_{2} \in E^{s}, \\
\left\|\xi_{1}-x_{1}\right\| & \leq \tau^{k}\left\|T^{k}\left(\xi_{1}-x_{1}\right)\right\| \leq \tau^{k}\left\|T^{k}\left(x-x_{0}\right)\right\|_{0} \leq c_{1} \tau^{k}\left\|T^{k}\left(x-x_{0}\right)\right\| \\
& \leq c_{1} \tau^{k}\left(\left\|T^{k}(x-z)\right\|+\left\|T^{k}\left(z-x_{0}\right)\right\|\right) \leq 2 c_{1} \tau^{k} \varepsilon \longrightarrow 0 \quad(k \longrightarrow+\infty), \\
\left\|\eta_{0}-x_{2}\right\| & \leq \tau^{k}\left\|T^{k}\left(\eta_{0}-x_{2}\right)\right\| \leq 2 c_{1} \tau^{k} \longrightarrow 0 \quad(k \longrightarrow+\infty) .
\end{aligned}
$$

Therefore $x=x_{0}$. Thus Lemma 5.4 is proved.

Lemma 5.5. (1) There exists $\delta>0$, such that $X_{p}=B_{\eta}\left(X_{p}\right)$ holds for $0<\eta<\delta$ and $p \in$ $\operatorname{Per}(T)$. (2) If $p, q \in \operatorname{Per}(T)$, and $q \in X_{p}$, then $X_{q}=X_{p}$.

Proof. (1) Obviously, $X_{p} \subset B_{\eta}\left(X_{p}\right)$. In the following we will prove that $B_{\eta}\left(X_{p}\right) \subset X_{p}$.

Firstly, for $\varepsilon>0$ small enough and $0<\eta<\varepsilon$, let $x \in B_{\eta}\left(X_{p}\right) \cap \operatorname{Per}(T)$. Since $X_{p} \subset$ $B_{\eta}\left(W_{p}\right) \subset B_{\eta}\left(X_{p}\right)$, there exists $\omega \in W_{p}$, such that $\|x-\omega\|<\eta<\varepsilon$. By Lemma 5.4, there exists a unique point $y$ such that $y \in W_{\varepsilon}^{u}(\omega) \cap W_{\varepsilon}^{s}(x)$. Thus $y \in W_{\varepsilon}^{u}(\omega) \cap E \subset$ $W^{u}(\omega) \bigcap E \subset W^{u}(p) \cap E=W_{p}$ and $y \in W^{s}(x)$. Suppose the period of $x$ is $l$. Then for each $k \in \mathbb{N}$, we have $\left\|T^{k l} x-T^{k l} y\right\| \rightarrow 0(k \rightarrow+\infty)$. So $\left\|x-T^{k l} y\right\| \rightarrow 0(k \rightarrow+\infty), x=$ $\lim _{k \rightarrow \infty} T^{k l} y \in \overline{W_{p}}=X_{p}$. Thus $B_{\eta}\left(X_{p}\right) \cap \operatorname{Per}(T) \subset X_{p}$.

Secondly, we have $B_{\eta}\left(X_{p}\right)=\overline{B_{\eta}\left(X_{p}\right)} \subset \overline{B_{\eta}\left(X_{p}\right) \cap \overline{\operatorname{Per}(T)}} \subset \overline{B_{\eta}\left(X_{p}\right) \cap \operatorname{Per}(T)} \subset \overline{X_{p}}=X_{p}$. Then $X_{p}=B_{\eta}\left(X_{p}\right)$.

(2) Since $X_{p}$ is the invariant set of $T$, then for each $y \in X_{q}$, there exists $z \in X_{q}$ such that for each $l \in \mathbb{N}, y=T^{l m} z$, where $m$ is the period of periodic point $q$. Furthurmore, according to the fact that $\overline{W^{u}(q)}=X_{q}$, there exist $z_{i} \in W^{u}(q)$ such that $\lim _{i \rightarrow+\infty} z_{i}=$ $z$, and $y=\lim _{i \rightarrow+\infty} T^{l m} z_{i}$. Since $z_{i} \in W^{u}(q)$, there exist $n_{0} \in \mathbb{N}$ and some constant $\eta>$ 0 , such that when $n>n_{0},\left\|T^{n}\left(z_{i}-q\right)\right\|>\eta$ holds. If $l$ is large enough, such that $l m>$ $n_{0}$, then $\left\|T^{l m}\left(z_{i}-q\right)\right\|>\eta$. Then for any $n \in \mathbb{N},\left\|T^{n}\left(T^{\operatorname{lm}}\left(z_{i}-q\right)\right)\right\|>\eta$ holds, that is, 
$\left\|T^{n+l m} z_{i}-T^{n} q\right\|>\eta$. Then $\left\|T^{n}(y-q)\right\|=\left\|T^{n} y-T^{n} q\right\|=\| T^{n} y-T^{n+l m} z_{i}+T^{n+l m} z_{i}-$ $T^{n} q\|\geq\| T^{n+l m} z_{i}-T^{n} q\|-\| T^{n} y-T^{n+l m} z_{i}\|\geq \eta-\| T\left\|^{n}\right\| y-T^{l m} z_{i} \|>\eta$, and we have $y \in W_{\eta}^{u}(q)$. Therefore $X_{q} \subset W_{\eta}^{u}(q)$.

For $q \in X_{p}$, then $W_{\eta}^{u}(q) \subset B_{\eta}\left(X_{p}\right)=X_{p}$, thus $X_{q} \subset X_{p}$.

For $0<\eta<\delta$ and $y \in X_{p} \cap X_{q}=B_{\eta}\left(W_{p}\right) \bigcap X_{q}$, then there exists $x \in W_{p}$ such that $\|x-y\|<\eta$, so $x \in B_{\eta}\left(X_{q}\right)=X_{q}$. Suppose the periods of $p, q$ are $l$ and $m$, respectively. Note that $x \in X_{q}$ and $X_{q}$ is a closed invariant set of $T^{m}$, so $\lim _{k \rightarrow+\infty}\left\|T^{-k l m}(q-x)\right\|=0$ holds. In addition, $x \in W_{p}=W^{u}(p) \bigcap E$, so $\lim _{k \rightarrow \infty}\left\|p-T^{-k l m} x\right\|=0$ stands. Similarly, for arbitrary $z \in W_{p}, \lim _{k \rightarrow \infty}\left\|q-T^{-k l m} z\right\|=0$. Hence $z \in T^{k l m} X_{q}=X_{q}$. Then $W_{p} \subset X_{q}$ and $X_{p}=\overline{W_{p}} \subset X_{q}$. Similarly, we can get $X_{q} \subset X_{p}$. In conclusion, we get $X_{p}=X_{q}$.

Proof of Theorem 5.1. Because $X$ is separable, there exists a countable series $\left\{x_{i}\right\}_{i \in \mathbb{N}}$, which is dense in $X$. For each $x_{i}$, we construct an open ball $B\left(x_{i}\right)$, which centers at $x_{i}$ with radius $\varepsilon / 3\left(\varepsilon=\delta / 2\right.$, and $\delta$ is discussed in Lemma 5.5(1)), such that $E \subset \bigcup_{i \in \mathbb{N}} B\left(x_{i}\right)$. Now that $\overline{\operatorname{Per}(T)}=E$, there exist countable periodic points $p_{i}(i \in \mathbb{N})$ such that $p_{i} \in$ $B\left(x_{i}\right)$. Let $\eta=\varepsilon$, then $B\left(x_{i}\right) \subset B_{\eta}\left(p_{i}\right)$, thus $E \subset \bigcup_{i \in \mathbb{N}} B\left(x_{i}\right) \subset \bigcup_{i \in \mathbb{N}} B_{\eta}\left(p_{i}\right) \subset \bigcup_{i \in \mathbb{N}} B_{\eta}\left(X_{p_{i}}\right)$. By Lemma 5.5(1), we have $B_{\eta}\left(X_{p_{i}}\right)=X_{p_{i}}$, then $E \subset \bigcup_{i \in \mathbb{N}} X_{p_{i}}$. On the other hand, $X_{p_{i}}=$ $\overline{W^{u}\left(p_{i}\right) \cap E} \subset E, \bigcup_{i \in \mathbb{N}} X_{p_{i}} \subset E$, so $E=\bigcup_{i \in \mathbb{N}} X_{p_{i}}$. Obviously, we can suppose that any two of $X_{p_{i}}(i=1,2, \ldots)$ are disjoint (if not, let $X_{p_{i}}$ be the subtraction of the combination of the preceding sets from $\left.X_{p_{i}}\right)$. Note that $T W_{p_{i}}=W_{T p_{i}}, T X_{p_{i}}=X_{T p_{i}}$, so we can let $E_{i}$ be the combination of all $X_{p_{i}}$ wherein $p_{i}$ have the same period. Therefore we separate $E$ into the combination of closed sets $E_{i}(i=1,2, \ldots)$, and any two of $E_{i}(i=1,2, \ldots)$ are disjoint, that is, $E=\bigcup_{i \in \mathbb{N}} E_{i}$.

For any two different sets $X_{p_{i}}, X_{p_{j}} \subset E_{i}$, there exists $l \in \mathbb{N}$, such that $T^{l} X_{p_{i}}=X_{p_{j}}$. Now, in order to prove that for arbitrary nonempty open sets $U, V \subset E_{i}$, there exists $n \in \mathbb{N}$, such that $\left(T^{n} U\right) \cap V \neq \Phi$, we only have to prove that for arbitrary nonempty open sets $U, V \subset$ $X_{p_{i}} \subset E_{i}$, there exists $n \in \mathbb{N}$, such that $\left(T^{n} U\right) \cap V \neq \Phi$. In fact, since $\overline{\operatorname{Per}(T)}=E$, there exists a periodic point $q$ in $U \cap X_{p_{i}}$, then by Lemma 5.5(2), both $X_{q}=X_{p_{i}}$ and $V \cap X_{q}=$ $V \cap X_{p_{i}}=V \neq \Phi$ hold. Therefore, there exists $x \in V \cap X_{q}$. Suppose that the period of $q$ is $m$, then $\lim _{k \rightarrow+\infty}\left\|T^{-k m} x-q\right\|=\lim _{k \rightarrow+\infty}\left\|T^{-k m} x-T^{-k m} q\right\|=0, \lim _{k \rightarrow+\infty} T^{-k m} x=q \in$ $U$. Since $q$ is an inner point in open set $U$, then for constant $k$ large enough, $T^{-k m} x \in U$ stands. Then $x \in T^{k m} U \cap V$. Let $n=k m$, thus $\left(T^{n} U\right) \cap V \neq \Phi$. The proof is finished.

Remark 5.6. (1) $E_{i}$ in this theorem cannot be the second countable Baire set. Otherwise, $T$ is topologically transactive in $E_{i}$, that is, there exists a dense orbit of $T$ in $E_{i}$. Hence from [10], there exists a dense orbit of $T$ in $E$, that is, $T$ is a hypercyclic operator in $E$. This is contrary to Remark 4.3(2).

(2) Since $E_{i}$ in this theorem is not second countable Baire set, $T$ is impossibly hypercyclic in $E_{i}$.

\section{Local structural stability of nonwandering operators}

Structural stability is the key subject in the differentiable dynamical systems (see $[25,29$, $30,32,33,36])$. It is well known that hyperbolic linear shift operators can keep their hyperbolic invariant properties under small perturbation, which inspires us to make an attempt to study the local structural stability of the nonwandering operators. 
Let $(X,\|\cdot\|)$ be a Banach space, $\mathfrak{R}(X)=\{f \mid f: X \rightarrow X$ is a continuous linear mapping with $\left.\sup _{x \in X}\|f(x)\|<\infty\right\}$. Obviously, it is a Banach space with the norm $\|f\|_{\mathfrak{R}}=$ $\sup _{x \in X}\|f(x)\|$.

LEMMA 6.1. Let $T$ be an invertible nonwandering operator relative to closed subspace $E \subset$ $X, S_{1}, S_{2} \in \mathfrak{R}(E)$, which satisfy $\operatorname{Lip}\left(S_{1}\right), \operatorname{Lip}\left(S_{2}\right)<\min \left\{1-\tau,\left\|T^{-1}\right\|^{-1}\right\}$. Then $T+S_{1}$ and $T+S_{2}$ are mutually topologically conjugate.

Proof. We only need to verify that there exists a homeomorphism $H=I+A(A \in \mathfrak{R}(E))$ satisfying

$$
H \circ\left(T+S_{1}\right)=\left(T+S_{2}\right) \circ H
$$

By reducing (6.1), we have

$$
A \circ\left(T+S_{1}\right)=T \circ A+S_{2} \circ(I+A)-S_{1}
$$

Projecting (6.2) onto subspace $E^{u}, E^{s}$ then we can get

$$
\begin{aligned}
& A_{s} \circ\left(T+S_{1}\right)=T_{s} \circ A_{s}+S_{2 s} \circ(I+A)-S_{1 s}, \\
& A_{u} \circ\left(T+S_{1}\right)=T_{u} \circ A_{u}+S_{2 u} \circ(I+A)-S_{1 u},
\end{aligned}
$$

where $A_{s}=P_{s} A, A_{u}=P_{u} A, T_{s}=P_{s} T, T_{u}=P_{u} T, S_{i s}=P_{s} S_{i}, S_{i u}=P_{u} S_{i}, P_{s}, P_{u}$ are, respectively, the projective operators from $E$ onto subspaces $E^{s}, E^{u}$.

For all $T \in \mathfrak{R}(E), x \in E, x=x_{s}+x_{u}, x_{s} \in E^{s}, x_{u} \in E^{u}, T x=T_{s} x+T_{u} x$, thus we have $T_{s} x=T_{s} x_{s}=T x_{s} \in E^{s}, T_{u} x=T_{u} x_{u}=T x_{u} \in E^{u}$.

Take equivalent norm $\|T\|_{\mathfrak{R}}=\sup _{x \in E}\|T x\|=\sup _{x \in E}\left\{\left\|T_{s} x\right\|,\left\|T_{u} x\right\|\right\}=\max \left\{\left\|T_{s}\right\|_{\mathfrak{R}}\right.$, $\left.\left\|T_{u}\right\|_{\Re}\right\}$.

Due to $\operatorname{Lip}\left(S_{1}\right)<\left\|T^{-1}\right\|^{-1}, T+S_{1}$ is invertible and also a Lipschitz mapping. By the reversibility of map $T_{u}=\left.T\right|_{E_{u}}$, we modify (6.3) and have

$$
\begin{aligned}
& A_{s}=T_{s} A_{s}\left(T+S_{1}\right)^{-1}+S_{2 s}(I+A)\left(T+S_{1}\right)^{-1}-S_{1 s}\left(T+S_{1}\right)^{-1}, \\
& A_{u}=-T_{u}^{-1}\left[S_{2 u}(I+A)-S_{1 u}-A_{u}\left(T+S_{1}\right)\right] .
\end{aligned}
$$

Now we write the right part of $(6.4)$ as $\Gamma_{s}(A), \Gamma_{u}(A)$, then

$$
\begin{aligned}
\left\|\Gamma_{s}(\eta)-\Gamma_{s}(\xi)\right\|_{\mathfrak{R}} & \leq\left(\tau+\operatorname{Lip}\left(S_{2}\right)\right)\|\eta-\xi\|_{\mathfrak{R}}, \\
\left\|\Gamma_{u}(\eta)-\Gamma_{u}(\xi)\right\|_{\mathfrak{R}} & \leq \tau\left(1+\operatorname{Lip}\left(S_{2}\right)\right)\|\eta-\xi\|_{\mathfrak{R}}, \quad \forall \eta, \xi \in \mathfrak{R}(E) .
\end{aligned}
$$

Obviously, the norms of $\left\|\Gamma_{s}\right\|$ and $\left\|\Gamma_{u}\right\|$ are no more than $\tau+\operatorname{Lip}\left(S_{2}\right)$.

Define $\Gamma: \mathfrak{R}(E) \rightarrow \mathfrak{R}(E), \Gamma(A)=\Gamma_{s}(A)+\Gamma_{u}(A)$, and $\|\Gamma\|_{\mathfrak{R}}=\max \left\{\left\|\Gamma_{s}\right\|_{\mathfrak{K}},\left\|\Gamma_{u}\right\|_{\mathfrak{R}}\right\}$, then $\|\Gamma(\eta)-\Gamma(\xi)\|_{\Re} \leq\left(\tau+\operatorname{Lip}\left(S_{2}\right)\right)\|\eta-\xi\|_{\Re}$, for all $\eta, \xi \in \mathfrak{R}(E)$. So $\Gamma$ is a contractive mapping. By the Banach contraction mapping principle, there exists a unique map $A \in \mathfrak{R}(E)$, such that

$$
(I+A) \circ\left(T+S_{1}\right)=\left(T+S_{2}\right) \circ(I+A)
$$


3906 Nonwandering operators in Banach space

Reciprocating $S_{1}, S_{2}$, we can get a unique map $P \in \mathfrak{R}(E)$, such that

$$
(I+P) \circ\left(T+S_{2}\right)=\left(T+S_{1}\right) \circ(I+P) .
$$

From (6.6) and (6.7), we deduce

$$
(I+P)(I+A)\left(T+S_{1}\right)=\left(T+S_{1}\right)(I+P)(I+A),
$$

or

$$
(I+\mathbb{Q})\left(T+S_{1}\right)=\left(T+S_{1}\right)(I+\mathbb{Q}), \quad \text { where } \mathbb{Q}=A+P(I+A) .
$$

In the operator equation (6.8), $\mathbb{Q}=0 \in \mathfrak{R}(E)$ is unique, so $(I+P)(I+A)=I$.

Similarly, $(I+A)(I+P)=I$ holds, then $H=I+A: E \rightarrow E$ is a homeomorphism. Therefore, $T+S_{1}$ and $T+S_{2}$ are mutually topologically conjugate.

Remark 6.2. The unique solution $H=I+A$ satisfying (6.1) only exists in the field of $A \in \mathfrak{R}(E)$. Otherwise, we can obtain solutions $I+0(0 \in \mathfrak{R}(E))$ and $I+I(I \notin \mathfrak{R}(E))$ when $S_{1}=S_{2}=0$.

Theorem 6.3. Let $T$ be an invertible nonwandering operator relative to closed subspace $E \subset X$, then $T$ is locally structurally stable.

Proof. Choosing a neighborhood $U_{\varepsilon}(T)$ of $T$ in $L(X)$ as $U_{\varepsilon}(T)=\{S \mid S \in B(X)$, $\| S-$ $T \| \leq \varepsilon\}$, where $0<\varepsilon<(1 / 2) \min \left\{1-\tau,\left\|T^{-1}\right\|^{-1}\right\}$.

Let

$$
S_{1}(x)= \begin{cases}(S-T)(x), & \|x\| \leq r \\ (S-T)\left(\frac{r x}{\|x\|}\right), & \|x\| \geq r\end{cases}
$$

$(r>0)$, then it is easily established that $S_{1} \in \mathfrak{R}(E)$, and $\operatorname{Lip}\left(S_{1}\right) \leq 2\|S-T\| \leq \varepsilon<\min$ $\left\{1-\tau,\left\|T^{-1}\right\|^{-1}\right\}$. Moreover, if $V=\{x \in E \mid\|x\|<r\}$, we get $T+\left.S_{1}\right|_{V}=S$. Considering special case of Lemma 6.1, that is, $S_{2}=0$, we know that $T$ and $S$ are topologically conjugate on $V$, thus $T$ is locally structurally stable.

\section{Acknowledgments}

Research was supported by the National Nature Science Foundation of China (10071033) and Natural Science Foundation of Jiangsu Province (BK2002003).

\section{References}

[1] S. I. Ansari, Hypercyclic and cyclic vectors, J. Funct. Anal. 128 (1995), no. 2, 374-383.

[2] Existence of hypercyclic operators on topological vector spaces, J. Funct. Anal. 148 (1997), no. 2, 384-390.

[3] J. Banks, J. Brooks, G. Cairns, G. Davis, and P. Stacey, On Devaney's definition of chaos, Amer. Math. Monthly 99 (1992), no. 4, 332-334.

[4] L. Bernal-González, On hypercyclic operators on Banach spaces, Proc. Amer. Math. Soc. 127 (1999), no. 4, 1003-1010. 
[5] J. P. Bès, Invariant manifolds of hypercyclic vectors for the real scalar case, Proc. Amer. Math. Soc. 127 (1999), no. 6, 1801-1804.

[6] J. P. Bès and A. Peris, Hereditarily hypercyclic operators, J. Funct. Anal. 167 (1999), no. 1, 94112.

[7] G. D. Birkhoff, Démonstration d'un théorème élémentaire sur les fonctions entières, C. R. Acad. Sci. Paris 189 (1929), 473-475.

[8] J. Bonet and A. Peris, Hypercyclic operators on non-normable Fréchet spaces, J. Funct. Anal. 159 (1998), no. 2, 587-595.

[9] P. S. Bourdon, Invariant manifolds of hypercyclic vectors, Proc. Amer. Math. Soc. 118 (1993), no. 3, 845-847.

[10] P. S. Bourdon and N. S. Feldman, Somewhere dense orbits are everywhere dense, Indiana Univ. Math. J. 52 (2003), no. 3, 811-819.

[11] K. C. Chan and J. H. Shapiro, The cyclic behavior of translation operators on Hilbert spaces of entire functions, Indiana Univ. Math. J. 40 (1991), no. 4, 1421-1449.

[12] R. deLaubenfels and H. Emamirad, Chaos for functions of discrete and continuous weighted shift operators, Ergodic Theory Dynam. Systems 21 (2001), no. 5, 1411-1427.

[13] R. L. Devaney, An Introduction to Chaotic Dynamical Systems, 2nd ed., Addison-Wesley Studies in Nonlinearity, Addison-Wesley, California, 1989.

[14] D. A. Dubin and M. A. Hennings, Quantum Mechanics, Algebras and Distributions, Pitman Research Notes in Mathematics Series, vol. 238, Longman Scientific \& Technical, Harlow; John Wiley \& Sons, New York, 1990.

[15] X.-C. Fu and J. Duan, Infinite-dimensional linear dynamical systems with chaoticity, J. Nonlinear Sci. 9 (1999), no. 2, 197-211.

[16] G. Godefroy and J. H. Shapiro, Operators with dense, invariant, cyclic vector manifolds, J. Funct. Anal. 98 (1991), no. 2, 229-269.

[17] M. González, F. León-Saavedra, and A. Montes-Rodríguez, Semi-Fredholm theory: hypercyclic and supercyclic subspaces, Proc. London Math. Soc. (3) 81 (2000), no. 1, 169-189.

[18] K.-G. Grosse-Erdmann, Universal families and hypercyclic operators, Bull. Amer. Math. Soc. (N.S.) 36 (1999), no. 3, 345-381.

[19] _ Hypercyclic and chaotic weighted shifts, Studia Mathematica 139 (2000), no. 1, 47-68.

[20] A. Gulisashvili and C. R. MacCluer, Linear chaos in the unforced quantum harmonic oscillator, J. Dyn. Syst. Meas. Control 118 (1996), no. 2, 337-338.

[21] J. K. Hale, Asymptotic Behavior of Dissipative Systems, Mathematical Surveys and Monographs, vol. 25, American Mathematical Society, Rhode Island, 1988.

[22] D. A. Herrero, Limits of hypercyclic and supercyclic operators, J. Funct. Anal. 99 (1991), no. 1, 179-190.

[23] , Hypercyclic operators and chaos, J. Operator Theory 28 (1992), no. 1, 93-103.

[24] C. Kitai, Invariant closed sets for linear operators, Ph.D. thesis, University of Toronto, Ontario, 1982.

[25] S. T. Liao, On the stability conjecture, Chinese Ann. Math. 1 (1980), no. 1, 9-30.

[26] C. R. MacCluer, Chaos in linear distributed systems, J. Dyn. Syst. Meas. Control 114 (1992), no. 2, 322-324.

[27] F. Martínez-Giménez and A. Peris, Chaos for backward shift operators, Internat. J. Bifur. Chaos Appl. Sci. Engrg. 12 (2002), no. 8, 1703-1715.

[28] E. Ott, Chaos in Dynamical Systems, Cambridge University Press, Cambridge, 1993.

[29] J. Palis and S. Smale, Structural stability theorems, Global Analysis (Proc. Sympos. Pure Math., Vol. 14, Berkeley, Calif, 1968), American Mathematical Society, Rhode Island, 1970, pp. 223-231.

[30] M. M. Peixoto, Structural stability on two-dimensional manifolds, Topology 1 (1962), no. 2, $101-120$. 


\section{Nonwandering operators in Banach space}

[31] V. Protopopescu and Y. Y. Azmy, Topological chaos for a class of linear models, Math. Models Methods Appl. Sci. 2 (1992), no. 1, 79-90.

[32] J. W. Robbin, A structural stability theorem, Ann. of Math. (2) 94 (1971), no. 3, 447-493.

[33] C. Robinson, Structural stability of $C^{1}$ diffeomorphisms, J. Differential Equations 22 (1976), no. $1,28-73$.

[34] S. Rolewicz, On orbits of elements, Studia Math. 32 (1969), 17-22.

[35] H. N. Salas, Hypercyclic weighted shifts, Trans. Amer. Math. Soc. 347 (1995), no. 3, 993-1004.

[36] S. Smale, The $\Omega$-stability theorem, Global Analysis (Proc. Sympos. Pure Math., Vol. 14, Berkeley, Calif, 1968), American Mathematical Society, Rhode Island, 1970, pp. 289-297.

[37] Z. Zhang, The Theory of Differentiable Dynamical System, Science Press, Beijing, 1997.

Lixin Tian: Nonlinear Scientific Research Center, Faculty of Science, Jiangsu University, Zhenjiang, Jiangsu 212013, China

E-mail address: tianlx@ujs.edu.cn

Jiangbo Zhou: Nonlinear Scientific Research Center, Faculty of Science, Jiangsu University, Zhenjiang, Jiangsu 212013, China

E-mail address: zjb76@ujs.edu.cn

Xun Liu: Nonlinear Scientific Research Center, Faculty of Science, Jiangsu University, Zhenjiang, Jiangsu 212013, China

E-mail address: liuxundy@163.com

Guangsheng Zhong: Nonlinear Scientific Research Center, Faculty of Science, Jiangsu University, Zhenjiang, Jiangsu 212013, China

E-mail address: zgs917@ntu.edu.cn 


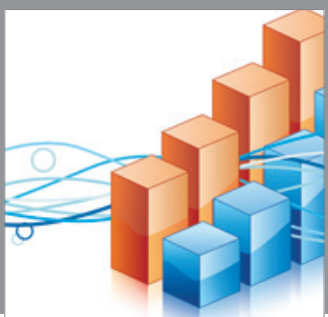

Advances in

Operations Research

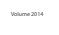

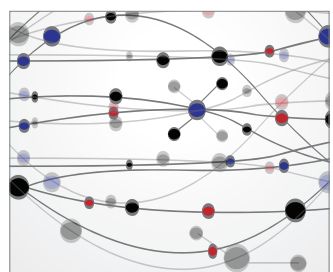

\section{The Scientific} World Journal
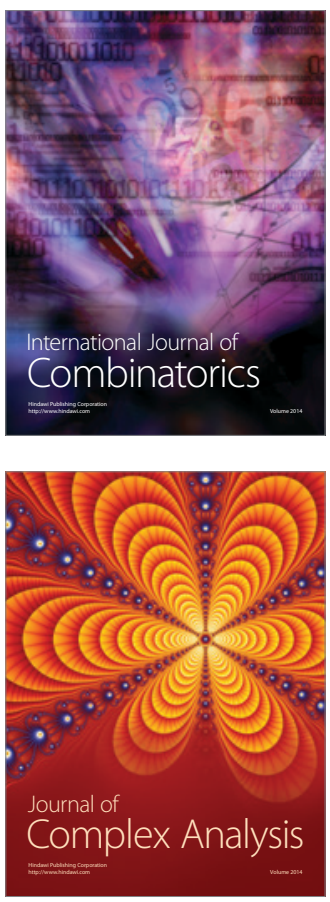

International Journal of

Mathematics and

Mathematical

Sciences
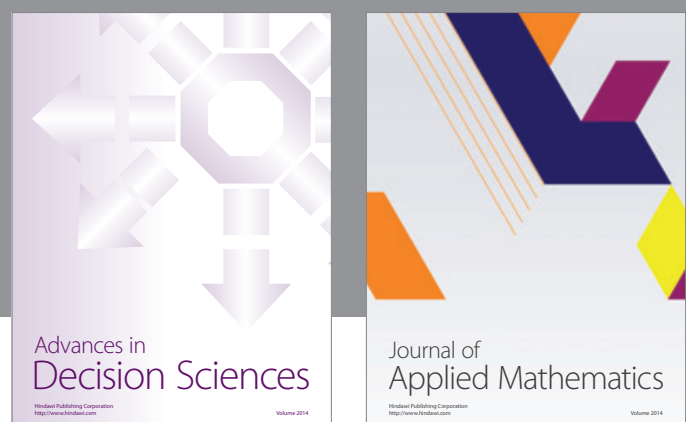

Journal of

Applied Mathematics
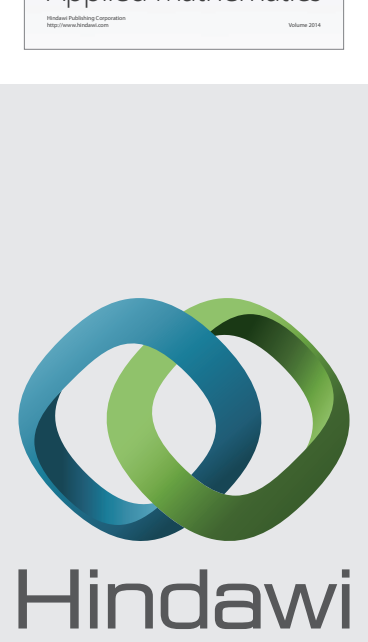

Submit your manuscripts at http://www.hindawi.com
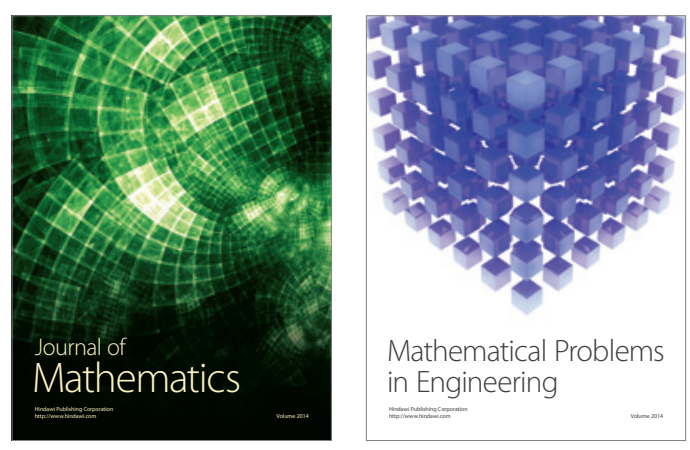

Mathematical Problems in Engineering
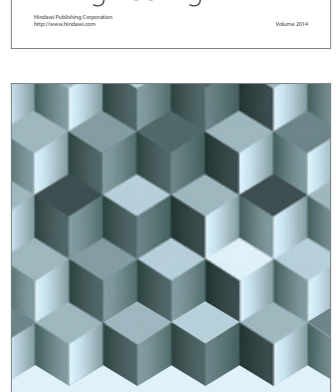

Journal of

Function Spaces
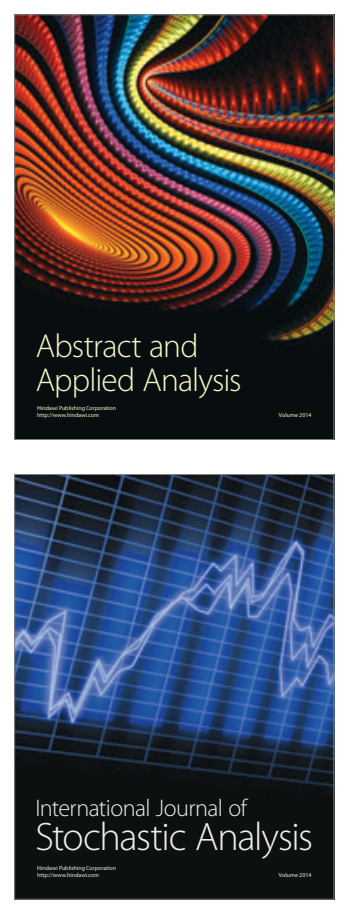

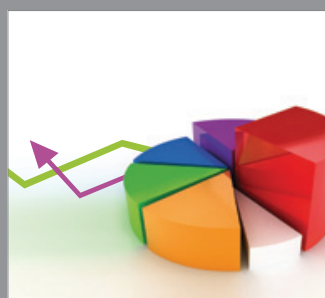

ournal of

Probability and Statistics

Promensencen
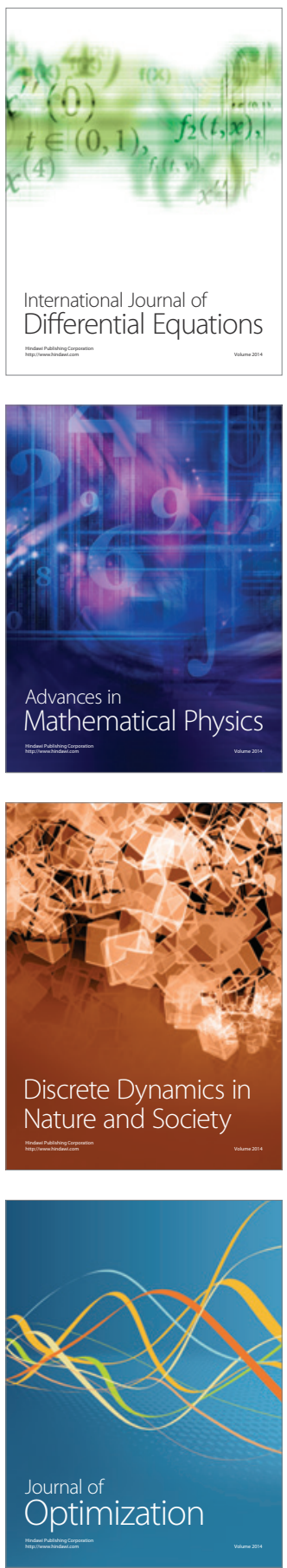